\title{
Relationship between achieved blood pressure, dietary habits and cardiovascular disease in hypertensive patients treated with olmesartan: the OMEGA study
}

Tamio Teramoto, Ryuzo Kawamori, Shigeru Miyazaki, Satoshi Teramukai, Masayuki Shirayama, Katsutoshi Hiramatsu, Fumiaki Kobayashi and the OMEGA Study Group

Hypertension Research (2012) 35, 1203; doi:10.1038/hr.2012.176

Correction to: Hypertension Research (2012) 35, 1136-1144; doi:10.1038/hr.2012.93; published online 5 July 2012

After the publication of this article, the authors noticed an error in the list of hospitals and institutions participated in this study, which was published as a Supplementary Information accompanying to this article.

The error has now been rectified and the corrected list is now available for download. 